\title{
MS03-P04 | CRYSTAL STRUCTURE OF AN ANTI-TUMOUR ANTIBODY IN COMPLEX WITH A
}

\section{TUMOUR-SPECIFIC ANTIGEN}

Johannesen, Hedda (University of Oslo, Department of Chemistry, Oslo, NOR); Bjerregaard-Andersen, Kaare (Department of Chemistry - University of Oslo, Oslo, NOR); Heggelund, Julie (Department of Pharmacy - University of Oslo, Oslo, NOR); Løset, Geir Åge (Department of Biosciences - University of Oslo, Oslo, NOR); Krengel, Ute (Department of Chemistry - University of Oslo, Oslo, NOR)

When a healthy cell mutates into a cancerous cell, it exhibits altered gene expression and uncontrolled proliferation. These changes can be exploited bycancer immunotherapy for diagnostic and therapeutic purposes. One such tumour-specific antigen is the $\mathrm{N}$-glycolyl GM3 ganglioside that is highly similar to the most common ganglioside $\mathrm{N}$-acetyl GM3, differing only by a terminal hydroxyl group instead of a hydrogen. It is located on the cell surface of several different tumours, but not in healthy adult human cells [3] The ganglioside is recognised by a monoclonal IgG antibody named 14F7 that was developed at the Centre for Molecular Immunology in Havana, Cuba [2]. To be able to exploit the favourable properties of 14F7 it would be helpful to know precisely how the $N$ glycolyl GM3 ganglioside and the 14F7 antibody interact at a molecular level. To this end, we have generated recombinant $14 \mathrm{F7}$ in scFv format, expressed the protein using a periplasmic expression system and crystallised the scFv [1]. Here, we show how the ganglioside binds to a groove created by the heavy chain CDR H3-loop.

[1] Bjerregaard-Andersen, K., Johannesen,et al., (2018). Crystal structure of an L chain optimised 14F7 antiganglioside Fv suggests a unique tumour-specificity through an unusual H-chain CDR3 architecture. Scientific Reports, 8(1), 10836.

[2] Carr, A., et al., (2000). A mouse IgG1 monoclonal antibody specific for $N$-glycolyl GM3 ganglioside recognized breast and melanoma tumors. Hybridoma, 19(3), 241-247.

[3] Malykh, Y. N. et al., (2001). N-Glycolylneuraminic acid in human tumours. Biochimie, 83(7), 623-634. 